\title{
Combining electrospinning and cell sheet technology for the development of a multiscale tissue engineered ligament construct (TELC)
}

\author{
Cedryck Vaquette, ${ }^{1}$ P. T. Sudheesh Kumar, ${ }^{2}$ Eugen Bogdan Petcu, ${ }^{2,3}$ Saso Ivanovski ${ }^{2}$ \\ ${ }^{1}$ Queensland University of Technology (QUT), Brisbane, Australia \\ ${ }^{2}$ Menzies Health Institute Queensland, Griffith University, Gold Coast, Queensland 4222, Australia \\ ${ }^{3}$ School of Medicine, Griffith University, Gold Coast, Queensland 4222, Australia
}

Received 22 August 2016; revised 17 November 2016; accepted 26 November 2016

Published online 00 Month 2017 in Wiley Online Library (wileyonlinelibrary.com). DOI: 10.1002/jbm.b.33828

\begin{abstract}
Ligament tissue rupture is a common sport injury. Although current treatment modalities can achieve appropriate reconstruction of the damaged ligament, they present significant drawbacks, mostly related to reduced tissue availability and pain associated with tissue harvesting. Stem cell based tissue regeneration combined with electrospun scaffolds represents a novel treatment method for torn ligaments. In this study, a low fiber density polycaprolactone (PCL) electrospun mesh and sheep mesenchymal stem cells (sMSCs) were used to develop tissue engineered ligament construct (TELC) in vitro. The assembly of the TELC was based on the spontaneous capacity of the cells to organize themselves into a cell sheet once seeded onto the electrospun mesh. The cell sheet matured over 4 weeks and strongly integrated with the low fiber density electrospun mesh which was subsequently processed into a ligament-like bundle and braided with two other bundles to develop the final construct. Live/dead assay revealed that the handling of the
\end{abstract}

construct through the various phases of assembly did not cause significant difference in viability compared to the control. Mechanical evaluation demonstrated that the incorporation of the cell sheet into the braided construct resulted in significantly modifying the mechanical behavior. A stress/displacement J-curve was observed for the TELC that was similar to native ligament, whereas this particular feature was not observed in the non-cellularized specimens. The regenerative potential of the TELC was evaluated ectopically in immunocompromized rats, compared to non cellularized electrospun fiber mesh and this demonstrated that the TELC was well colonized by host cells and that a significant remodelling of the implanted construct was observed. ๑ 2017 Wiley Periodicals, Inc. J Biomed Mater Res Part B: Appl Biomater 00B: 000-000, 2017.

Key Words: electrospinning, cell sheet, anterior cruciate ligament, braiding, tissue remodelling, tissue engineering

How to cite this article: Vaquette C, Sudheesh Kumar PT, Petcu EB, Ivanovski S. 2017. Combining electrospinning and cell sheet technology for the development of a multiscale tissue engineered ligament construct (TELC). J Biomed Mater Res Part B 2017:00B:000-000.

\section{INTRODUCTION}

Anterior cruciate ligament is a connective tissue which plays an important role in knee stability and to prevent relative movement between two bones. The anterior cruciate ligament (ACL) possesses a complex anatomy including fibrocartilage regions at both the femoral insertion site and in the vicinity of the tibial attachment site, as well as an interconnecting soft tissue connective region in the intraarticular portion of the tissue. Although it is essentially composed of an aligned network of collagen type I and III fibrils into which spindle-shaped ligament fibroblasts are inserted, chondrocyte-like cells can be found in the distal fibrocartilaginous regions. ${ }^{1}$ The vascularization of the ligament is mainly provided by the geniculate artery which branches out and covers the ligament. The ligament vascularization is however heterogeneous and the bony attachment sites along with the anterior fibrocartilaginous portion are completely avascular. ${ }^{1,2}$ The ACL withstands a complex pattern of mechanical loading ranging from tensile, torsional and compressional forces each in specific locations of the tissue. ${ }^{3,4}$ During normal walking, the physiological load applied to the ACL is in the range of several hundred Newtons ${ }^{5,6}$ whereas the ultimate tensile strength is around $2000 N^{7}$ Because of the range of mechanical loading, especially in pivotal solicitation, ACL tearing is one of the most common sport injuries, essentially affecting a young and active population. The conjunction of heterogeneous vascular distribution throughout the tissue and the presence of several fibrocartilaginous zones significantly contribute to the poor healing capacity of the ACL.

Current treatments commonly utilize either autologous graft tissue or synthetic implants. Autologous graft using 
Patellar Tendon Bone ${ }^{8}$ or Semitendinous Gracilis Tendon ${ }^{9}$ are the gold standards of ACL replacement and have been proven to be efficient and safe reconstructive strategies. Drawbacks of the utilization of autologous tissues originate from grafting material availability, tissue morbidity and pain related to tissue harvesting. On the other hand, synthetic implants alleviate some of the aforementioned limitations, but despite recent reports of positive short to mid-term clinical outcomes they are still associated with poor long term stability, risk of osteolysis and synovitis. Recent developments in the optimization of these prosthetic devices have focused on the surface modification of the polymeric material for the purpose of enhancing the integration at the bony interfaces. ${ }^{10,11}$

Tissue engineering provides alternative approach to current treatments. It involves the combination of cells and biomaterial scaffolds in vitro to create implantable constructs that restore the function of the damaged tissue. To this end, a tissue engineered ligament construct can be developed in vitro using specific and well-controlled conditions. Amongst the plethora of constructs developed in recent years, the utilization of cell sheets offers the advantage of efficiently delivering both cells and more importantly, an intact extracellular matrix capable of initiating or contributing to the subsequent tissue regeneration. Indeed, the utilization of cell sheets enables the implantation of an already welldeveloped tissue constructs with an extensive collagenous network. ${ }^{12,13}$ The concept of cell sheet engineering has been popularized by the emergence of smart surfaces allowing cell detachment utilizing the hydrophilic/hydrophobic transition of the substrate. ${ }^{14}$ This avoids the use of chemicals, which are potentially harmful to cells and detrimental to the ECM. Another more cost-effective method of preparing cell sheets utilizes spontaneous cell sheet contraction combined with the application of a small force to harvest the sheet ${ }^{15-17}$ without notably affecting cell viability. Even though the cell sheets are sufficiently robust to withstand mechanical harvesting, a carrier is usually required to facilitate the handling and surgical placement of the resulting construct.

The present study reports on the physical and biological assessment of a novel low fiber density electrospun mesh combined with cell sheet technology for potential application in ligament tissue engineering.

\section{MATERIALS AND METHODS}

\section{Electrospun mesh manufacturing and characterization}

Electrospun meshes were utilized for manufacturing a braided construct. To this end, medical grade polycaprolactone (PCL) (80 kDa, Purac, The Netherlands) was dissolved in a mixture of chloroform/dimethylformamide (Merck, Australia) (9:1) at a concentration of $25 \%(\mathrm{w} / \mathrm{v})$. The polymer solution was placed in a $10-\mathrm{mL}$ glass syringe fitted with a 23 gauge blunt needle and electrospun at a flow rate of 2 $\mathrm{mL} \mathrm{h}^{-1}$, an applied voltage of $10 \mathrm{kV}$ and a spinneret to collector distance of $20 \mathrm{~cm}$. Electrospinning was performed on a $9 \times 9 \mathrm{~cm}^{2}$ aluminum foil collector for $30 \mathrm{~s}$. Thereafter the electrospun membrane was transferred into a $14.6 \mathrm{~cm}$ diameter petri dish (Thermo Fisher Scientific, Australia) and sterilized by a $30 \mathrm{~min}$ ethanol immersion followed by a 30 min UV exposure in a biosafety cabinet. After complete evaporation of the ethanol, the electrospun mesh remained bonded by electrostatic interactions to the surface of the plastic petri dish.

For morphological characterization, the electrospun membrane was fixed on carbon tape attached to a metallic stub and sputter coated with gold. The membranes were imaged using SEM (Quanta 200, USA). The pore area distribution was evaluated using ImageJ software. Briefly, the SEM images were imported into the software and the greyscale threshold of each image was then adjusted to remove the background and obtained a white and black image. Thereafter the area occupied by each pore was measured using an already available algorithm according to a published method ${ }^{18}$. One image of three different membranes at a magnification of 500 times was used to calculate the pore area and an average of the obtained data was used to determine the pore size distribution. The fiber diameter was also noted from the SEM images by the measurement tool using imageJ software. Here again, three images from three different electrospun meshes were utilized and around 20 fibers were measured.

\section{Stem cell isolation and expansion}

Bone marrow mesenchymal stem cells (bMSCs) were obtained from the iliac crest of a 5-year-old female merino sheep. The cells were isolated by selective adhesion onto plastic culture plates ${ }^{19}$ and utilized at passage 3 .

\section{Assembly of the tissue engineered ligament construct (TELC)}

The electrospun meshes were seeded with $1 \times 10^{6}$ cells (passage 3 ) in $1 \mathrm{~mL}$ of medium and care was taken to evenly distribute the cellular suspension on the electrospun fibers. The cells were allowed to attach to the mesh for $3 \mathrm{~h}$ and $15 \mathrm{~mL}$ DMEM (high glucose with 10\% FBS and 1\% penicillin/streptomycin) with ascorbic acid (50 $\mu \mathrm{g} \mathrm{mL}^{-1}$ ) was subsequently added to fully cover the membrane. Thereafter, particular attention was paid to the handling of the Petri dishes in order to prevent any detachment of the electrospun mesh caused by excessive motion of the culture media. The medium was changed every 3 days and the ascorbic acid contributed to the formation of a mature cell sheet extending onto the entire surface of the dish 4 weeks post-seeding which encapsulated the electrospun mesh [Figure 1(A-D)]. The portions of the cell sheet located outside the area covered by the electrospun fibers were delicately handled and folded over the mesh which was subsequently rolled using two sterile forceps into a tubular structure resembling a ligament bundle as shown in Figure 1(E,F). This was repeated for another two meshes and the ends of the three bundles were attached together using a suture (Prolene 4.0). The bundles were subsequently braided in order to create a tissue engineered ligament construct (TELC) as depicted in Figure $1(\mathrm{G}, \mathrm{H})$. The TELC was further incubated overnight at $37^{\circ} \mathrm{C}$ and thereafter used for subsequent evaluation and characterization. 

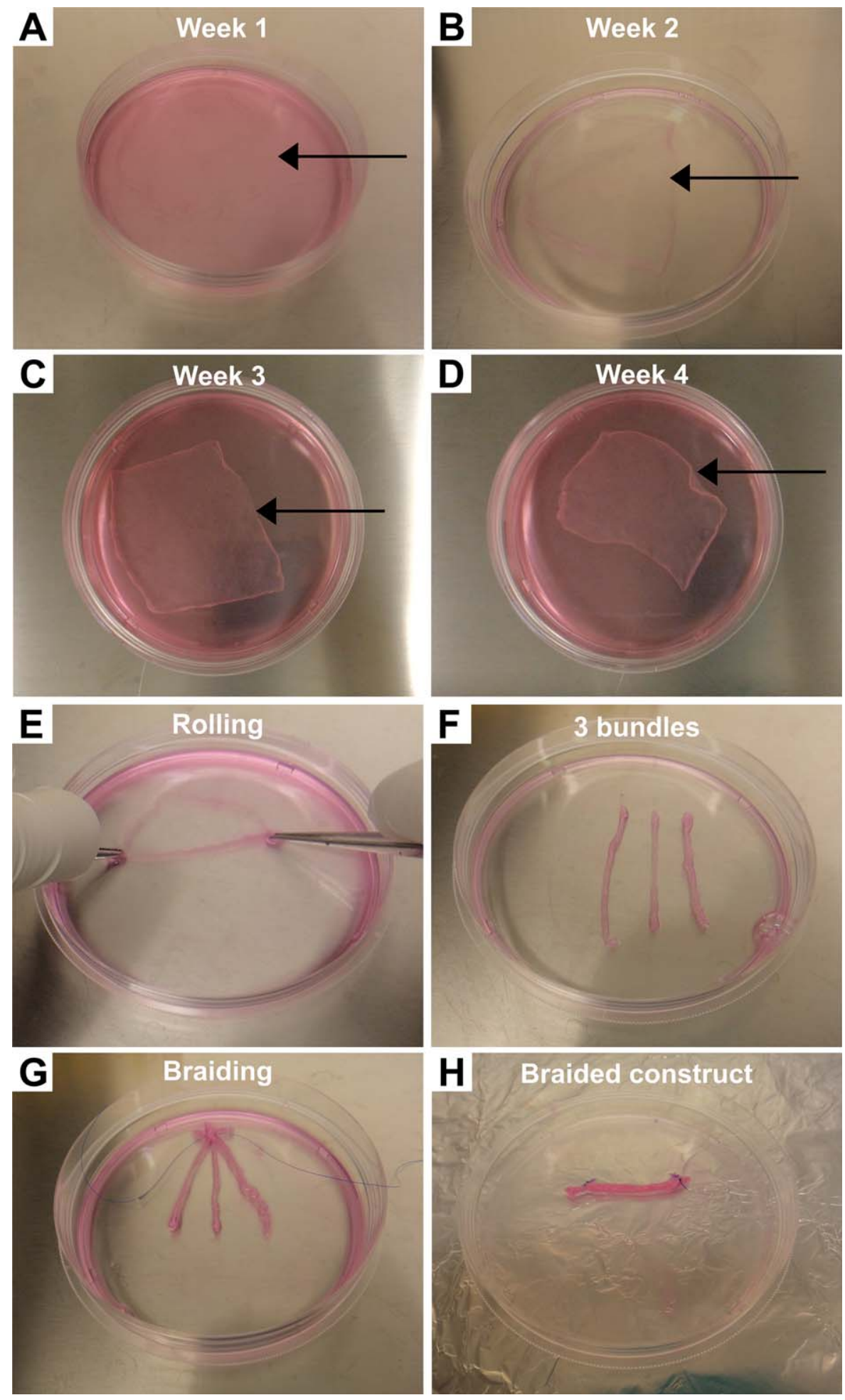

FIGURE 1. (A-D) Cells cultured on electrospun membrane for A: 1 week, B: 2 weeks, C: 3 weeks, D: 4 weeks. Arrows point to the edges of the cell encapsulated membrane. (E) Creation of cylindrical cellularized scaffold (bundles) by rolling the electrospun mesh after 4 weeks of culture. (F) Aligning three bundles for subsequent braiding. G) Suturing of three scaffolds together prior to braiding the construct. (H) Braided Tissue Engineered Ligament Construct (TELC).

The porosity of the non-cellularized construct was assessed in order to evaluate the changes experienced during the post-processing (rolling and braiding). This was determined using three different samples by liquid displacement method using ethanol. ${ }^{20}$ Briefly, a known volume (V1) of absolute ethanol was taken in a graduated cylinder and the braided scaffolds were immersed in it and kept for $5 \mathrm{~min}$. The total volume of ethanol and ethanol penetrated scaffold was 
noted as V2. After $5 \mathrm{~min}$, the scaffolds were removed from the cylinder and the volume of remaining ethanol was noted as V3. Three scaffolds were used for the experiment and an average of the data calculated. The percentage porosity was calculated using the following formula:

$$
\% \mathrm{P}=\frac{(\mathrm{V} 1-\mathrm{V} 3)}{(\mathrm{V} 2-\mathrm{V} 3)}
$$

\section{In vitro characterization}

Cell viability evaluation based on live/dead assay. Cell viability of the TELC ( $\sim 12 \mathrm{~h}$ post braiding) and control electrospun mesh (cellularized electrospun scaffolds not processed into a tubular structure) was assessed using live/ dead assay. The samples were washed twice with phosphate buffered saline (PBS) and immersed in a solution of Fluorescein diacetate (FDA, Excitation/Emission 495/520 nm) at $5 \mu \mathrm{g} \mathrm{mL}^{-1}$ and Propidium iodide (PI, Excitation/Emission were $540 / 608 \mathrm{~nm}$ ) at $2 \mu \mathrm{g} \mathrm{mL}^{-1}$ for $10 \mathrm{~min}$ in the incubator. Both FDA and PI were purchased from Sigma-Aldrich, USA. The samples were then washed with PBS and imaged using a confocal laser microscope (Nikon A1R+, USA). Three different braided and flat scaffolds with cells were used and imaged to quantify cell viability. Image s software was used to quantify the cell viability based on the red color intensity of dead cells and green color intensity of viable cells.

Histology and scanning electron microscopy. Histology was utilized for investigating the morphology of the TELC. To this end, the TELC were paraffin embedded and sectioned $(4 \mu \mathrm{m})$ using a microtome (Leica) for Hematoxylin and Eosin (H \&E) and Van Gieson staining in order to visualize the tissue morphology and the collagen deposition. The corresponding braided control scaffold (braided electrospun mesh without cells) was not investigated as PCL is solubilized during the sample processing. For SEM imaging, the TELC was fixed with $4 \%$ paraformaldehyde for $1 \mathrm{~h}$ and washed three times with PBS. The dried TELC was sputter coated with gold and imaged using SEM.

\section{Mechanical testing}

The mechanical properties of the TELC were tested after 4 weeks of 2D in vitro culture, following an overnight incubation post-braiding Tensile test of TELC (as measured $\sim 12 \mathrm{~h}$ post braiding, $40 \mathrm{~mm}$ long and $2 \mathrm{~mm}$ diameter) and control (40 $\mathrm{mm}$ long and $0.3 \mathrm{~mm}$ diameter) scaffolds were conducted using an Instron 5848 Microtester (Instron, USA) fitted with a $5 \mathrm{~N}$ load cell. The control scaffold had a smaller diameter as they did not contain any cellular material. Both control and TELC were immersed in media prior to the test. Each end ( $5 \mathrm{~mm}$ ) of TELC and control scaffolds were sandwiched in sand article and thereafter placed in between the grips of the micro tester. A $0.05 \mathrm{~N}$ preload was applied prior to starting the tensile test. The tensile test was performed in air at room temperature while the specimens were still hydrated, at a cross-head speed of $10 \mathrm{~mm} / \mathrm{min}$ with a $30 \mathrm{~mm}$ gauge length.
The stiffness was calculated from the linear section of the force/strain curve as previously reported ${ }^{21,22}$ and the ultimate force were also recorded. To assess statistical significance, six replicates for each group were utilized.

\section{In vivo evaluation}

The regenerative potential of the TELC was assessed in a subcutaneous athymic rat model. Griffith University animal ethics committee granted the approval for this study under ethics number DOH/04/15/AEC: "Ligament regeneration potential of cell laden polycaprolactone scaffold in immunocompromized rat." Three athymic nude male, 8-week-old rats (CBH-rnu/Arc rats, Animal resource center, Australia) were used for this in vivo study. Prophylactic antibiotic cover was provided prior to the surgery using subcutaneous injection of Keflin® (cefalotin sodium) $20 \mathrm{mg} \mathrm{kg}^{-1}$ and Gentamicin $5 \mathrm{mg} \mathrm{kg}^{-1}$. General anesthesia for all surgical procedures was administered with a Mediquip isoflurane vaporizer (dosage: $1-3 \%$ inhalant to effect, up to $5 \%$ for induction). The dorsal region of the rats were depilated and disinfected by application of chlorhexidine in alcohol solution. Four bilateral small incisions were made bilaterally and longitudinally along the central line of the shaved dorsal area and subcutaneous pockets were created with a pair of surgical scissors. The TELC (2 per rat) and control scaffolds ( 2 per rat) were implanted into the individual pockets. The skin was sutured using Prolene ${ }^{\circledR}$ suture 4-0 and the animal revival was assisted using pure oxygen. Figure 2(A) represents the control and TELC for the implantation. Figure 2(B-D) represents the ectopic site creation and subcutaneous implantation of control scaffold and TELC into athymic nude rats. Four weeks post-implantation, the animals were euthanized by isoflurane overdose and cervical dislocation and the constructs were retrieved and immediately fixed in $4 \%$ paraformaldehyde (PFA) solution for 2 days. The samples were then processed, paraffin embedded, sectioned (4 $\mu \mathrm{m}$ ) using a microtome and stained with $\mathrm{H} \& \mathrm{E}$ and Van Gieson as described in the previous section.

\section{Statistical analysis}

The data represented are \pm SD and are analyzed by $t$ test using GraphPad Prism ${ }^{\circledR}$ software. Data with $p$ values $<0.05$ indicate statistically significant.

\section{RESULTS}

\section{Fabrication and characterization of the electrospun membrane}

The morphology of the electrospun membranes was imaged using SEM [Figure 3(A)]. It consisted of a fibrous nonwoven mesh of electrospun material composed of only a few layer of fibers deposited on top of each other. This created a low fiber density electrospun mesh possessing a porous and interconnected network with fiber diameters ranging 1-4 $\mu \mathrm{m}$ and pore area ranging from $50-5000 \mu \mathrm{m}^{2}$ [Figure 3(B)]. Pore size distribution was further evaluated using ImageJ software and it was found that $>50 \%$ of the pores had an area of $100-500$ $\mu \mathrm{m} .{ }^{2}$ The electrospinning conditions combined with a short deposition time resulted in the manufacturing of a two 

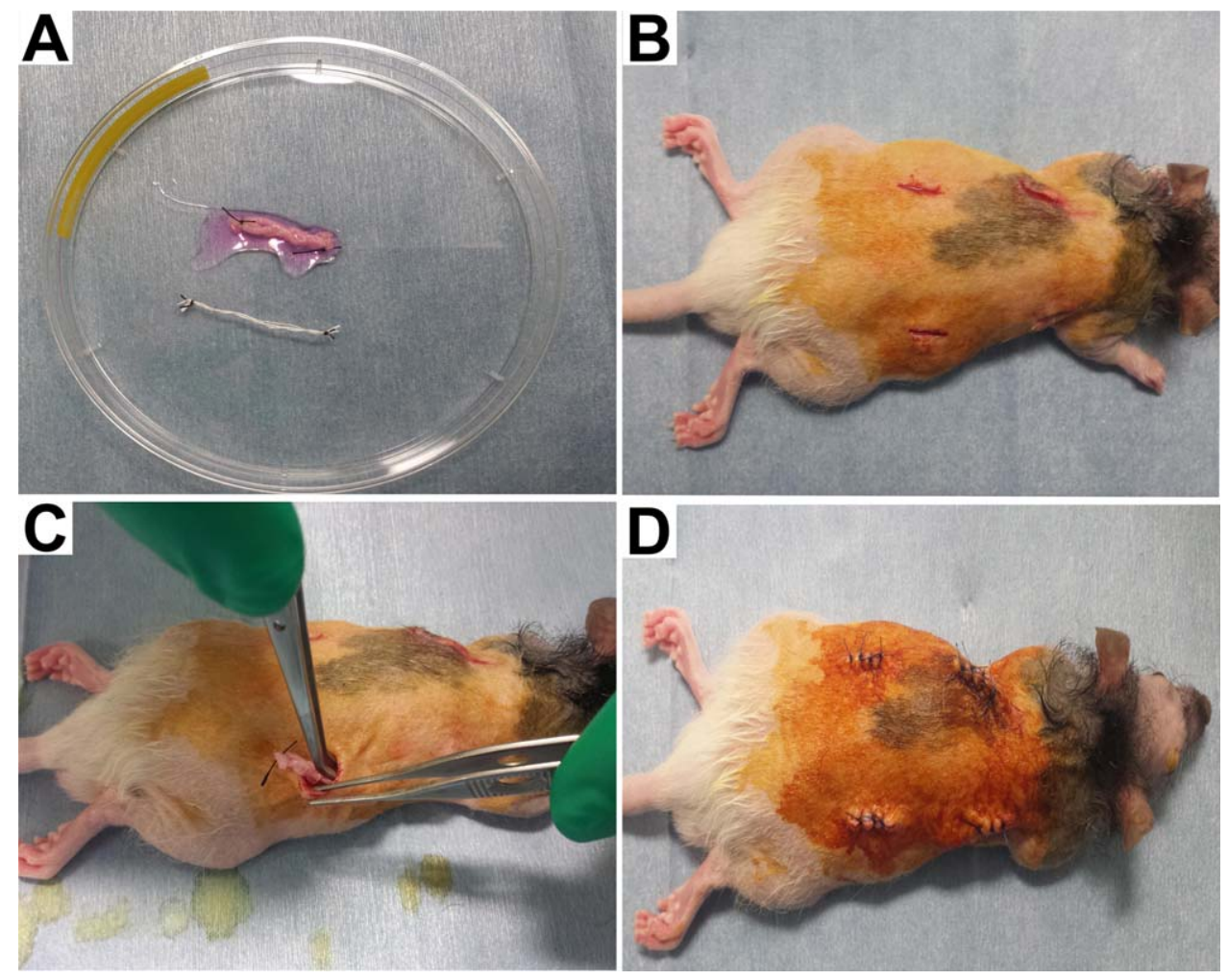

FIGURE 2. (A) Control and TELC (B) subcutaneous pocket creation (C) construct implantation and (D) surgical pockets were sutured.

dimensional (2D) low fiber density mesh suitable for subsequent cell colonization.

\section{TELC fabrication}

The fabrication of the TELC involved three main steps: (1) cell seeding and cell sheet formation, (2) creation of the bundles, (3) braiding of the bundles. In step (1), the cells were cultured for 4 weeks in order to enable the development of a cell sheet which could handle subsequent rolling and braiding. To achieve this, ascorbic acid was essential for ensuring efficient collagen crosslinking resulting in the formation of a robust cell sheet. Although, the cells were seeded on the low fiber density electrospun mesh only, they gradually colonized the entire petri dish as shown in Figure 1(A-D) and after 4 weeks of in vitro culture a continuous and mature cell sheet was observed in the culture dish [indicated by black arrows in Figure 1(A-D)]. The cell sheet had also encapsulated the electrospun fibers and incorporated them into the ECM, thus providing excellent cohesion and physical integrity necessary for the subsequent rolling step. The ligament-like bundles were assembled by applying rotational movements onto two corners of the electrospun mesh. This small mechanical load was sufficient to detach the cell sheet/electrospun mesh from the substrate and enabled the creation of a cylindrical cellularized electrospun scaffold [Figure 1(E)]. The assembly of the
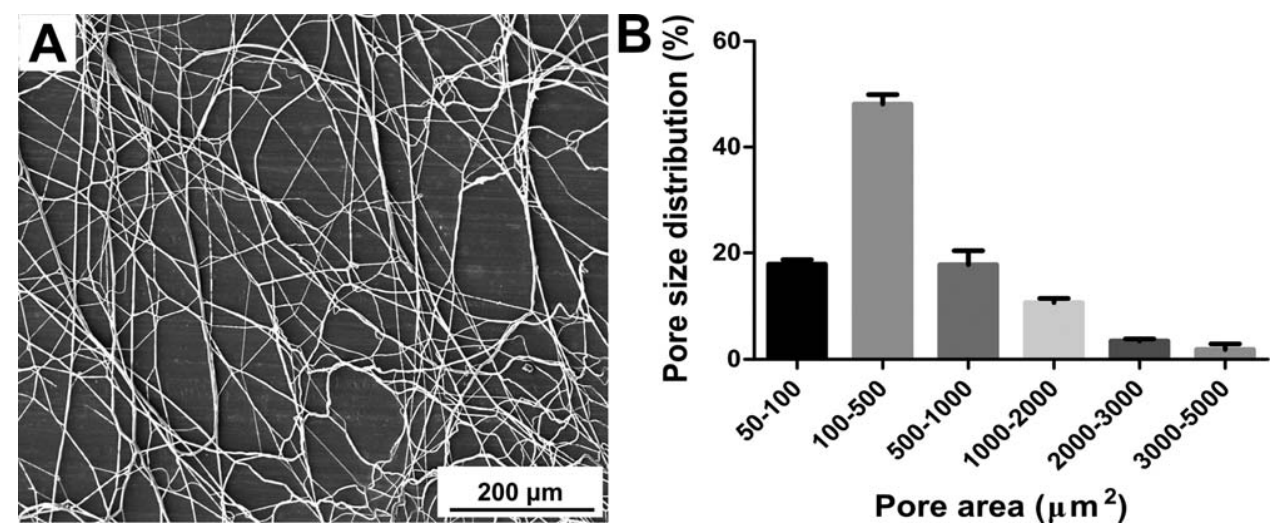

FIGURE 3. (A) SEM image of the electrospun membrane and (B) Pore area distribution. 

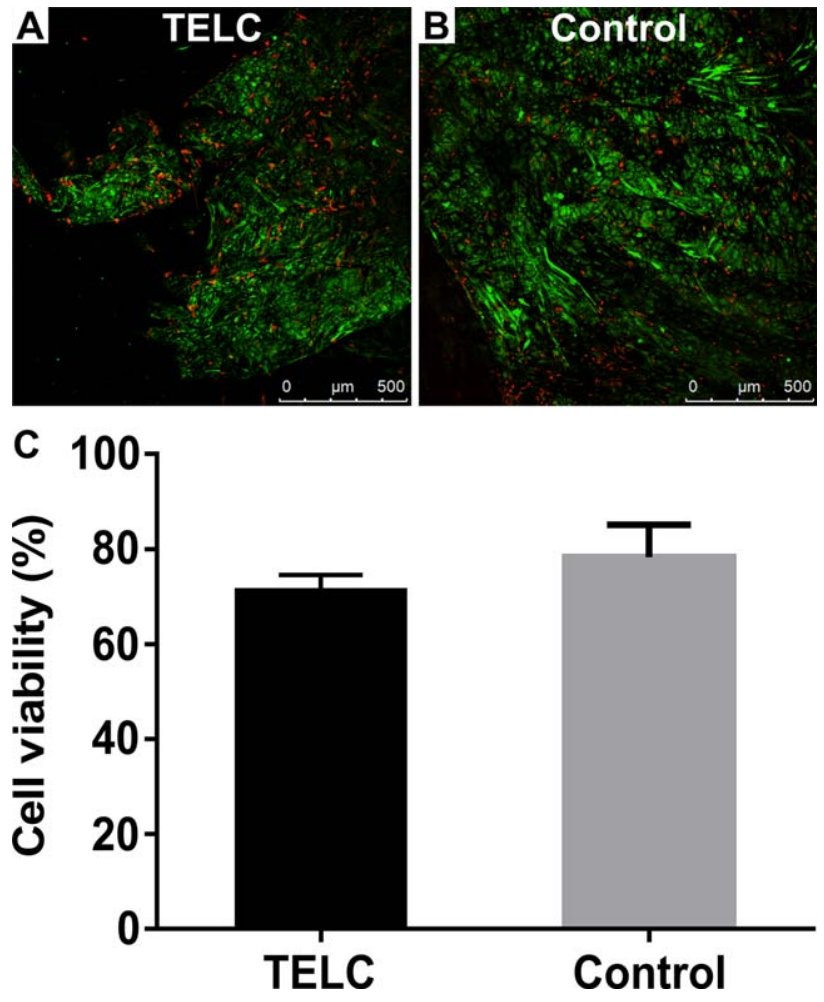

FIGURE 4. Live/dead assay confocal microscope image of (A) braided as measured $12 \mathrm{~h}$ post-braiding and (B) control unprocessed (2D) scaffold, for both groups the cells were cultured for 4 weeks. Red color indicates dead cells whereas green represents live cells. (C) Cell viability quantification using ImageJ software.

TELC was further performed by manually braiding three bundles together [Figure $1(F)$ ]. This was achieved by suturing together the three ends of each bundle as shown in Figure $1(G)$ and surgical forceps were utilized to position the bundle on the edge of the Petri dish. This resulted in the application of a slight mechanical load onto the bundles which was necessary for the braiding of the bundles. Once assembled, the TELC was immersed in media overnight in order to ensure an appropriate fusion of the bundles [Figure $1(\mathrm{H})]$.

\section{Cell viability evaluation of TELC by live/dead assay}

As the assembly of the TELC required the handling of the electrospun mesh combined with the application of a small but not inconsequential mechanical loading, the viability of the cells in the resulting construct was assessed using a live/dead assay. Figure $4(A, B)$ showed that there were no significant changes in the cell viability \% when comparing the TELC and the control cellularized electrospun mesh. The quantification of the percentage cell viability from live/dead assay images using imageJ software confirmed that the processing of the cellularized electrospun mesh did not significantly impact on the cell viability [Figure 4(C)].

\section{SEM and histology}

Figure 5(A-E) represents SEM images of control and TELC respectively. The TELC was thicker than the control scaffold due the presence of cellular component on the external portion of the braided bundles as the cell sheet has encapsulated the electrospun material. Figure 5(C) demonstrated that post-processing, (i.e., rolling and braiding), of the electrospun mesh resulted in some level of pore collapse. However, the pore size remained in the range of several tenths of microns which is in the lower rage for allowing cell infiltration and tissue colonization. In addition, the control samples experienced significant stretching resulting in alignment of the electrospun fibers as shown in Figure 5(B). The morphology of the TELC specimens revealed that the cell sheet had thoroughly encapsulated the electrospun fibers [square arrow head Figure 5(D)]. While the internal porous architecture of the electrospun fibers could not be seen using this imaging technique, it was hypothetized that the TELC experienced less pore collapse and less fibers stretching due to the presence of the cellular and ECM components (black arrow). Figure 5(E-L) depicts the H\&E and Van Gieson's staining of longitudinal and cross-sections of the TELC, demonstrating a homogeneous distribution of the ECM and cells within the electrospun bundles with the presence of collagen matrix intercalated between the electrospun fibers.

\section{Mechanical testing}

The mechanical properties of the TECL were assessed and compared to a non-cellularized braided electrospun mesh. Figure $6(\mathrm{~A}, \mathrm{~B})$ represent mechanical testing data of the control PCL scaffold and TELC, respectively. The control specimens displayed a typical behavior of porous polymeric materials under tensile load, featuring an initial linear relationship between force and strain until the material yielded and finally ruptured [Figure 6(A)]. The TELC displayed a totally different behavior with an initial deformation without significant increase in the force until 10\% strain, followed by an increase in the force as the specimen was further stretched. This resulted in a so called J-curve, typical of the response to tensile loading of ligamentous and tendinous tissues. These drastic changes in the deformation behavior also translated in major variations in the other mechanical parameters: the TELC was significantly softer than the control [Figure $6(\mathrm{C})$ ] and the force at failure was also significantly reduced for the TELCs compared to the control scaffold [Figure 6(D)].

\section{In vivo evaluation}

Upon sample retrieval 4 weeks post-implantation, an obvious difference was noted between the non-cellularized and cellularized scaffolds. The dimensions of the latter were significantly bigger and the detailed morphology of the electrospun fibers was not visible anymore as opposed to the control group in which the fibrous nature of the braided scaffold was still noticeable [Figure $7(\mathrm{~A}, \mathrm{~B})]$. Both groups displayed a white appearance with numerous blood vessels (black arrows) in the direct vicinity of the implanted constructs.

Histological analysis revealed that there were significant differences in regards to the cellular colonization of the scaffold, with the control scaffold [Figure $7(\mathrm{C}-\mathrm{E})$ ] displaying large portions of non-cellularized area (indicated by the stars) whereas 


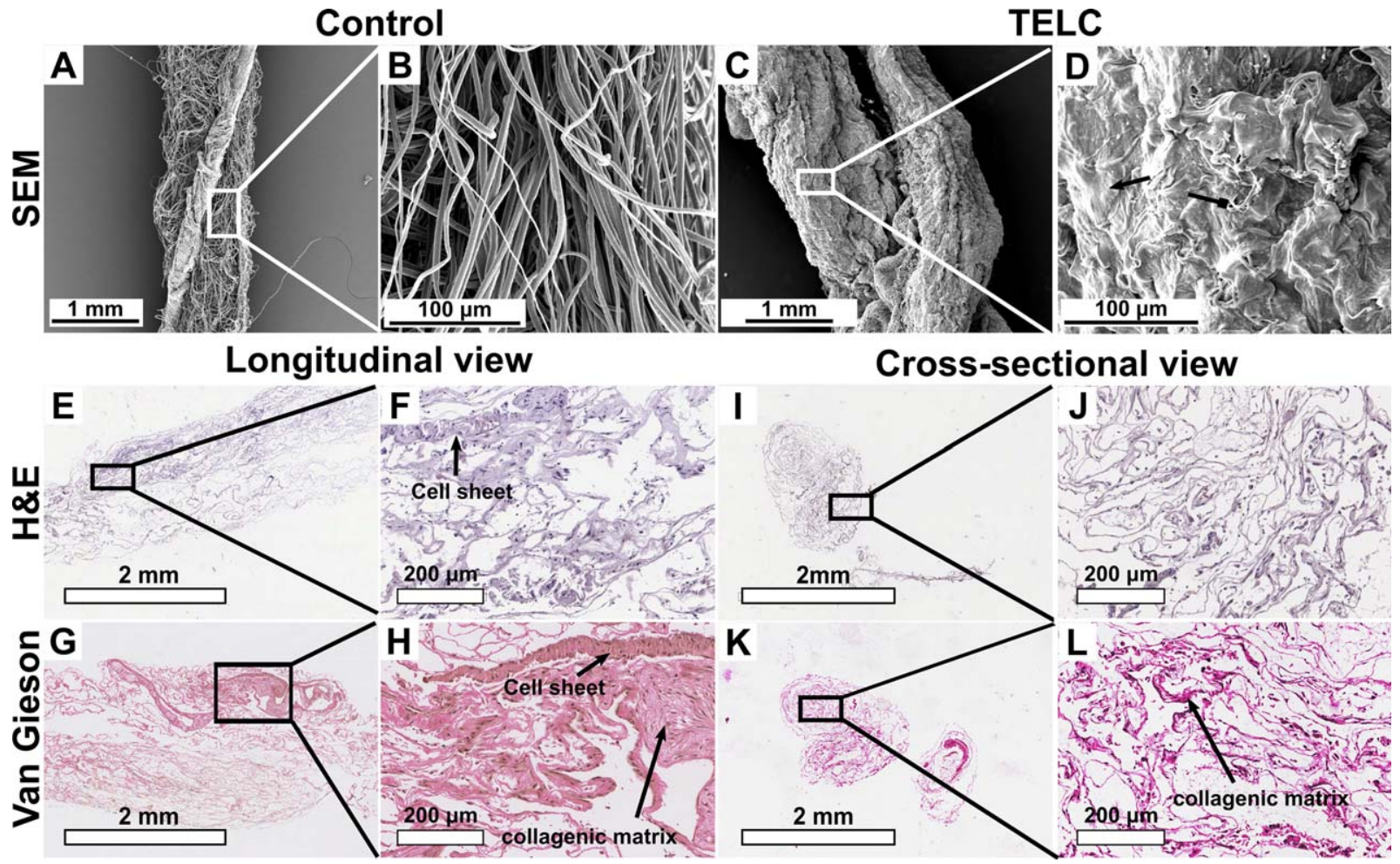

FIGURE 5. (A and B) SEM images of control (non-cellularized) braided scaffold, ( $C$ and D) SEM images of TELC. Square arrow head indicates the electrospun fibers which are embedded into the cell sheet (black arrow). (E, F) and (G,-H) representative H\&E and Van Gieson stained images of the TELC's longitudinal section demonstrating the homogeneous cell distribution and the deposition of collagen in between the electrospun fibers (note that the PCL electrospun fibers were dissolved during the processing for histology and hence cannot be seen in the images). $(\mathrm{I}, \mathrm{J})$ and $(\mathrm{K}, \mathrm{L})$ feature the $\mathrm{H} \& \mathrm{E}$ and Van Gieson staining of the TELC cross-section confirming the homogeneous cell distribution and the presence of an extensive collagenic matrix.

these areas were infrequent in the TELC [Figure $7(\mathrm{~L}-\mathrm{N})]$. These poorly cellularized portions were characterized by the presence of numerous electropsun fibers (white square arrow head), indicating that the changes in pore size and porosity (caused by the rolling and braiding) were detrimental to cell colonization in vivo [Figure $7(\mathrm{D}, \mathrm{E}, \mathrm{J}, \mathrm{K})]$. Collagenic tissue (fb) as seen per Van Gieson staining was observed in the direct surroundings and to some extents into the TELC [Figure $7(\mathrm{~L}, \mathrm{~N})$ ]
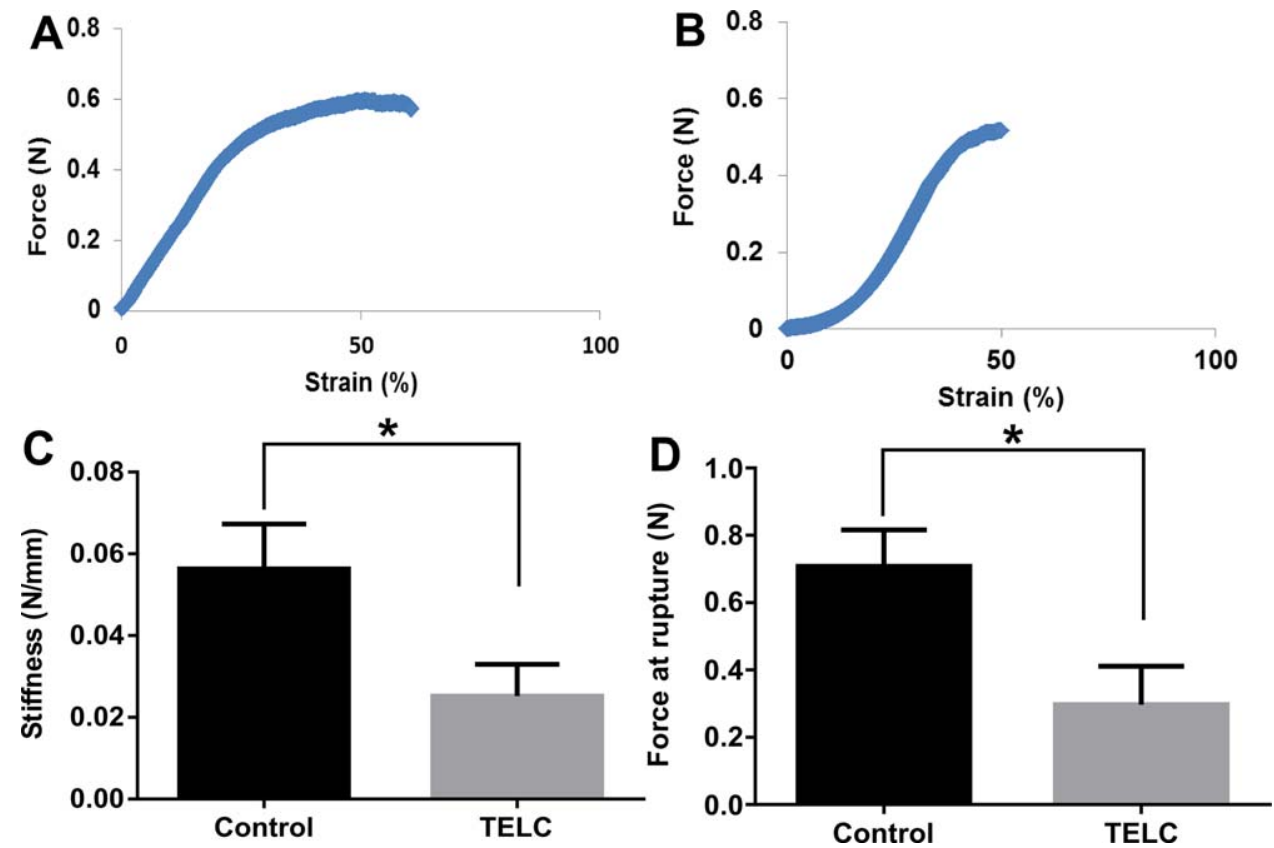

FIGURE 6. Tensile strength of (A) control (B) TELC (C) Stiffness of control and TELC (D) Force at failure of control and TELC. 

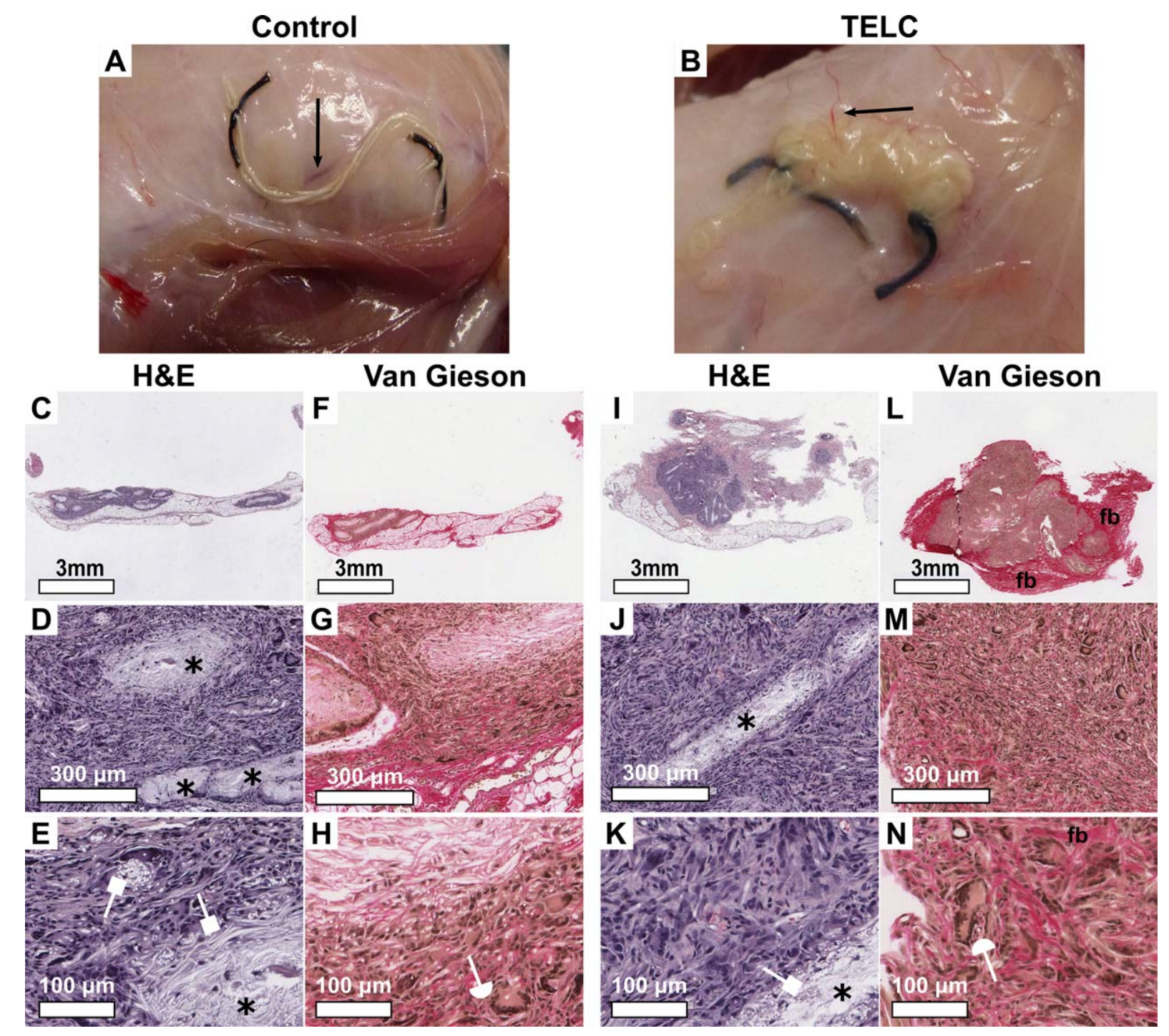

FIGURE 7. Explanted specimen 4 weeks post-implantation of (A) control (non-cellularized) (B) TELC, black arrows indicate the presence of vasculature in the vicinity of the construct. (C-E) representative H\&E images of control braided scaffold demonstrating the presence of numerous non-cellularized areas (as shown by *) which were closely interlinked with the concentration of electrospun fibers (white square arrow head). (FH) Van Gieson staining of the control braided scaffold showing a low level of collagen deposition and the presence of polynucleated cells (white semi-circular arrow head). (I-K) H\&E sections of the TELC indicating less frequent acellular zones. (L-N) Van Gieson staining of the TECL demonstrating the presence of fibrous tissue and collagen deposition around and into the TELC (as indicated by fb).

whereas this feature was not present in the control specimens. Both groups, with and without cells, induced a host reaction leading to the infiltration of polynucleated cells as shown in Figure $7(\mathrm{H}, \mathrm{N})$ (semi-circular arrow head) which resulted in tissue remodelling leading to the removal of the implanted ECM and original cellular component of the TELC.

\section{DISCUSSION}

This proof of concept study investigated the utilization of cell sheet technology combined with a low fiber density electrospun carrier in order to create a multiscale tissue engineering ligament construct. The approach followed a bio-mimicking strategy whereby the structural components of the TECL are assembled from collagen fibers, secreted by the cells during the cell sheet formation, to ligament-like bundles, thereafter processed into a ligament like construct, hence recapitulating to some extent the architecture of the native tissue.

The manufacturing technique developed for assembling the TELC required significant manual handling hence the know-how of the operator was a parameter of importance for the successful and reproducible preparation of the constructs. Although, this can potentially induce operator to operator variation, it is consistent with current clinical practices whereby delicate manual preparation of explanted autologous tissues is performed prior to re-implantation. ${ }^{23,24}$ Extensive in vitro preparation and cell maturation is widely reported $^{15,22,25,26}$ for the purpose of creating or assembling a TELC and this also involved significant manual handling of cellularized constructs under aseptic conditions, similar to the present study. The assembly of the construct must be implemented in a manner ensuring that sterility and cell 
viability are not compromised throughout the handling of the constructs, as it has also been emphasized in previous studies. ${ }^{27,28}$ Several groups reported the utilization of cell sheets combined with various engineering systems, such as pins ${ }^{29}$ and U-shaped metallic devices ${ }^{22}$ in order to allow the gradual in vitro self-assembly of the engineered ligament. Contrarily to these approaches, in the present study the TELC utilized a two-step in vitro maturation approach: (1) collagen deposition allowing cell sheet maturation and (2) manual assembly of the TELC. Hence this involved mechanically detachment of the cell sheet once proper integration into the electrospun scaffold was achieved. This resulted in a slightly harsher processing method when compared to other in vitro self-assembly methods whereby small and constant mechanical loading is applied in order to mature the engineered construct. However the mechanical detachment of the cell sheet was not detrimental to cell viability as previously reported ${ }^{17}$ and permitted the rapid assembly of the TELC subsequent to its in vitro maturation.

Braiding is a well-established technology for the production of various products of high technicity and requiring high strength such as marine or climbing ropes. Braiding enables the enhancement of mechanical strength for a given material and hence is a technique of interest for the manufacturing of anterior cruciate ligament prostheses. The necessity of achieving high mechanical strength is however generally detrimental to cell and tissue infiltration due to small pore sizes and poor interconnection resulting from the braiding. ${ }^{30}$ Despite some recent advances in the design of braided constructs enabling the presence of pore size gradient throughout the construct, ${ }^{31,32}$ the tissue integration of braided scaffolds is generally impeded by the physical barrier created by the small pore dimension. The biomaterial carrier utilized in the present study was specifically designed to incorporate large pores and this was achieved by reducing the deposition time of the electrospun fibers. A low electrospun fiber density was essential for allowing the encapsulation of the electrospun fibers within the cell and the ECM secreted during the in vitro culture. The rolling of this cell sheet/carrier resulted in alternating cell layers of mature ECM and electrospun fibers, hence creating a homogenous cell distribution in the bundle and subsequently into the braided construct.

Unlike other highly vascularized tissue, ligament requires extended time in order to remodel and heal. Hence, the slow degradation profile imparted through the use of PCL provides physical and mechanical support within an adequate time frame for allowing initial regeneration and long term tissue remodeling before significant degradation and loss of mechanical properties. A late acute inflammatory response is also avoided with the use of PCL as the acidic degradation byproducts are gradually evacuated via a surface erosion mechanism, ${ }^{33}$ as opposed to bulk degradation leading to a burst release and significant local $\mathrm{pH}$ reduction, as observed with faster degrading polymers such as PLA and PGA.

Although, PCL displays appropriate biocompatibility and tissue integration, it lacks bioactivity, hence the incorporation of biological cues are necessary to initiate the regenerative process subsequent to implantation. This can be achieved through incorporation of growth factors into an electrospun mesh ${ }^{34}$ and by combining the scaffold with cells which is the strategy utilized in the present study. The concept of cell sheet technology has been pioneered and extensively utilized for tissue engineering and regenerative medicine by the prominent group of Prof. Okano. ${ }^{35}$ The utilization of a thermo-responsive surface for harvesting the cell sheet has become the gold standard, however harvesting can be also implemented with gentle mechanical stimulation $^{16,17}$ as described in the present study. Cell sheet technology offers many advantages over conventional cell delivery approaches. First it is easily implemented and circumvents many technical issues associated with homogenously distributing cells into a 3D construct with macroscopic pore size. This is not trivial and although several solutions have been implemented, mainly using fibrous biomaterials filler within the large pores of a given ligament scaffold, ${ }^{36-38}$ the culture of a large dimension cellularized construct remains problematic in regards to oxygen and nutrient diffusion to the central portion of the construct. Indeed, heterogeneous nutrient and oxygen supply within a large or dense construct can lead to poor in vitro tissue distribution and organization. Recently, a slightly different approach involving the insertion of ligament cell spheroids into a 3D construct was shown to enable the seeding of cells with an already developed ECM. However, homogenous distribution of the spheroids remained elusive. ${ }^{39}$ The utilization of a 2D system mitigated this later issue and ensured that the cells and the ECM were homogenously distributed throughout the braided TELC. Second, cell sheets are an efficient way of delivering cells and perhaps more importantly their intact ECM. The delivery of an intact ECM is generally achieved through the in vitro culture of cellularized 3D constructs with all of the aforementioned limitations (heterogeneous cell distribution, poor nutrient, and oxygen supply in the center of the construct and so forth). The implantation of an intact network of ECM is probably as important as the delivery of viable cells and has been shown to significantly promote tissue regeneration. ECM is a natural niche for binding growth factors and hence probably plays an important role in the chemotaxis subsequent to implantation leading to tissue regeneration.

Cell sheet technology is hence a promising methodology and has already been clinically trialed for several applications. ${ }^{35}$ Nevertheless it encompasses several disadvantages related to the intrinsic nature of the cell sheet. Indeed, the technology relies on the cells own ability to form sufficient ECM, and in this later respect, the use of ascorbic acid or other biochemicals stimulating the production of ECM was essential for developing a mature cell sheet. In addition, cell sheets are mechanically weak and care should be taken upon their handling and implantation. To achieve enhanced mechanical properties, the TELC developed in the present study utilizes a biomaterial carrier and this facilitated its handling.

The tensile strength of the TELC remained well below that of native tissue and it was also observed that the mechanical strength of the non-cellularized construct was 
significantly higher than the TELC. This tends to indicates than the cells and the prolonged immersion in the cell culture media have favored the diffusion of water molecules into the PCL network and hence either acted as a plasticizer. This resulted in a decrease in strength, stiffness and more positively in changing the mode of deformation which became similar to that of the native tissue. The shift in deformation behavior is likely caused by the stretching experienced in the control specimens that induced fiber alignment whereas the presence of cells and ECM components dampened this effect in the TELCs. Increasing the mechanical properties of the TELC in order to manufacture an implantable cellularized device capable of withstanding surgical handling and preloading upon graft placement remains a challenge. A dual bundle braiding technique utilizing six bundles (as opposed to three in the current method) would result in strengthening the TELC while increasing the overall dimension of the construct hence reaching the size of the native tissue. However this could cause significant drawbacks related to diffusion of nutrient and oxygen throughout the scaffold post-braiding. Noteworthy, the braided scaffold is not a continuous single cylindrical scaffold and hence the various bundles are exposed to the local environment over the major part of their surface area hence enabling nutrient and oxygen diffusion.

The last 3 decades of ligament prosthesis design has been driven by a "biomechanical mimicking approach" whereby the construct is required to reach high tensile strength prior to implantation, as used in commercially available approaches such as the Ligament Augmentation \& Reconstruction System (LARS) or at a research level. ${ }^{40,41}$ An emerging trend in ligament tissue engineering focusses on the development of tissue constructs with biological composition and collagen architecture similar to that of the native ligament. ${ }^{22,26,29,42-44}$ Common to these approaches is the in vitro maturation of a well-developed collagen ECM network leading to the selfassembly of the ligament construct. This is achieved using a biomaterial carrier which degrades within the time frame of the in vitro culture ${ }^{42}$ or in a scaffold-free approach whereby cells are allowed to spontaneously form ligament like tissue in vitro. The orientation of the collagenic network is obtained by applying a constant gentle mechanical loading onto the self-assembled tissue, hence further mimicking the organization of the collagen fibers occurring in the native tissue.

Although the mechanical properties of the self-assembled tissues are not systematically reported, several studies demonstrated that the tensile strength remained in the range of those measured in the present study, that is, below a few Newtons. ${ }^{42,43,45}$ Hence, in the context of ACL replacement where physiological loading is in the hundreds of Newtons during normal locomotion, these self-assembled constructs and the TELC developed in the present study do not seem to be biomechanically viable. However, Calve et al. ${ }^{45}$ demonstrated that the self-assembled constructs experienced significant remodelling once implanted in vivo in the rat groin. This resulted in increased collagen content, collagen fibril thickening and higher tensile stiffness. ${ }^{45}$ These authors further implemented this concept by performing ACL reconstruction in an ovine model. No failure of the implanted constructs was observed and more interestingly, an increase in mechanical properties was reported over time, reaching half of the native ligament tangential modulus. ${ }^{26}$ This exemplifies the significance of the remodelling events occurring during in vivo implantation, enabling the utilization of an initially weak tissue engineered constructs for ACL reconstruction such as the one developed in the present study.

\section{CONCLUSIONS}

This study reports the manufacture and characterization of tissue engineered ligament constructs consisting of braided cell sheet/electrospun PCL scaffold layers. Promising results were obtained in terms of homogeneity of cell/ECM distribution within the construct and tissue integration in vivo. The overall biomechanical strength of the construct remains a potential barrier to clinical use, and the effect of in vivo remodeling on the development of physiological biomechanical properties needs to be tested in clinically relevant models.

\section{REFERENCES}

1. Petersen W, Tillmann B. Structure and vascularization of the cruciate ligaments of the human knee joint. Anat Embryol (Berl) 1999;200:325-334.

2. Scapinelli R. Vascular anatomy of the human cruciate ligaments and surrounding structures. Clin Anat 1997;10:151-162.

3. Giori NJ, Beaupre GS, Carter DR. Cellular shape and pressure may mediate mechanical control of tissue composition in tendons. J Orthop Res 1993;11:581-591.

4. Castile RM, Skelley NW, Babaei B, Brophy RH, Lake SP. Microstructural properties and mechanics vary between bundles of the human anterior cruciate ligament during stress-relaxation. J Biomech 2016;49:87-93.

5. Morrison JB. The mechanics of the knee joint in relation to normal walking. J Biomech 1970;3:51-61.

6. Gabriel MT, Wong EK, Woo SL, Yagi M, Debski RE. Distribution of in situ forces in the anterior cruciate ligament in response to rotatory loads. J Orthop Res 2004;22:85-89.

7. Woo SL, Hollis JM, Adams DJ, Lyon RM, Takai S. Tensile properties of the human femur-anterior cruciate ligament-tibia complex. The effects of specimen age and orientation. Am J Sports Med 1991;19:217-225.

8. Alm A, Gillquist J, Stromberg B. The medial third of the patellar ligament in reconstruction of the anterior cruciate ligament. A clinical and histologic study by means of arthroscopy or arthrotomy. Acta Chir Scand Suppl 1974;445:5-14.

9. Sajovic M, Strahovnik A, Dernovsek MZ, Skaza K. Quality of life and clinical outcome comparison of semitendinosus and gracilis tendon versus patellar tendon autografts for anterior cruciate ligament reconstruction: An 11-year follow-up of a randomized controlled trial. Am J Sports Med 2011;39:2161-2169.

10. Li H, Chen S. Biomedical coatings on polyethylene terephthalate artificial ligaments. J Biomed Mater Res A 2015;103:839-845.

11. Vaquette C, Viateau V, Guerard S, Anagnostou F, Manassero M, Castner DG, Migonney V. The effect of polystyrene sodium sulfonate grafting on polyethylene terephthalate artificial ligaments on in vitro mineralisation and in vivo bone tissue integration. Biomaterials 2013;34:7048-7063.

12. Matsuda N, Shimizu T, Yamato $M$, Okano $T$. Tissue engineering based on cell sheet technology. Adv Mater 2007;19:3089-3099.

13. Yang J, Yamato $M$, Shimizu T, Sekine H, Ohashi K, Kanzaki M, Ohki T, Nishida K, Okano T. Reconstruction of functional tissues with cell sheet engineering. Biomaterials 2007;28:5033-5043.

14. Okano $T$, Yamada N, Sakai $H$, Sakurai $Y$. A novel recovery system for cultured cells using plasma-treated polystyrene dishes grafted 
with poly(N-isopropylacrylamide). J Biomed Mater Res 1993;27: 1243-1251.

15. Ouyang HW, Toh SL, Goh J, Tay TE, Moe K. Assembly of bone marrow stromal cell sheets with knitted poly (I-lactide) scaffold for engineering ligament analogs. J Biomed Mater Res B Appl Biomater 2005;75:264-271.

16. Costa PF, Vaquette $C$, Zhang $Q$, Reis RL, Ivanovski $S$, Hutmacher DW. Advanced tissue engineering scaffold design for regeneration of the complex hierarchical periodontal structure. J Clin Periodontol 2014;41:283-294.

17. Vaquette C, Fan W, Xiao Y, Hamlet S, Hutmacher DW, Ivanovski $\mathrm{S}$. A biphasic scaffold design combined with cell sheet technology for simultaneous regeneration of alveolar bone/periodontal ligament complex. Biomaterials 2012;33:5560-5573.

18. Hao L, Savalani MM, Zhang Y, Tanner KE, Heath RJ, Harris RA. Characterization of selective laser-sintered hydroxyapatite-based biocomposite structures for bone replacement. Proc R Soc A 2007;463:1857-1869.

19. Vaquette C, Slimani S, Kahn CJ, Tran N, Rahouadj R, Wang X. A poly(lactic-co-glycolic acid) knitted scaffold for tendon tissue engineering: $A n$ in vitro and in vivo study. J Biomater Sci Polym Ed 2010;21:1737-1760.

20. Guan J, Fujimoto KL, Sacksa MS, Wagner WR. Preparation and characterization of highly porous, biodegradable polyurethane scaffolds for soft tissue applications. Biomaterials 2005;26:3961-3971.

21. Liu H, Fan $H$, Wang $Y$, Toh SL, Goh JC-H. The interaction between a combined knitted silk scaffold and microporous silk sponge with human mesenchymal stem cells for ligament tissue engineering. Biomaterials 2008;29:662-674.

22. Ni M, Rui YF, Tan Q, Liu Y, Xu LL, Chan KM, Wang Y, Li G. Engineered scaffold-free tendon tissue produced by tendon-derived stem cells. Biomaterials 2013;34:2024-2037.

23. Brown $\mathrm{CH}$, Steiner ME, Carson EW. The use of hamstring tendons for anterior cruciate ligament reconstruction. Technique and results. Clin Sports Med 1993;12:723-756.

24. Muneta $T$, Koga $H$, Mochizuki T, Ju YJ, Hara K, Nimura A Yagishita K, Sekiya I. A prospective randomized study of 4-strand semitendinosus tendon anterior cruciate ligament reconstruction comparing single-bundle and double-bundle techniques. Arthroscopy 2007;23:618-628.

25. Fan $\mathrm{H}$, Liu $\mathrm{H}$, Toh $\mathrm{SL}$, Goh JC. Anterior cruciate ligament regeneration using mesenchymal stem cells and silk scaffold in large animal model. Biomaterials 2009;30:4967-4977.

26. Mahalingam VD, Behbahani-Nejad N, Horine SV, Olsen TJ, Smietana MJ, Wojtys EM, Wellik DM, Arruda EM, Larkin LM. Allogeneic versus autologous derived cell sources for use in engineered bone-ligament-bone grafts in sheep anterior cruciate ligament repair. Tissue Eng A 2015;21:1047-1054.

27. Spalazzi JP, Dagher E, Doty SB, Guo XE, Rodeo SA, Lu HH. In vivo evaluation of a multiphased scaffold designed for orthopaedic interface tissue engineering and soft tissue-to-bone integration. J Biomed Mater Res A 2008;86:1-12.

28. Thayer PS, Dimling AF, PlessI DS, Hahn MR, Guelcher SA Dahlgren LA, Goldstein AS. Cellularized cylindrical fiber/hydrogel composites for ligament tissue engineering. Biomacromolecules 2014;15:75-83.

29. Syed-Picard FN, Larkin LM, Shaw CM, Arruda EM. Three-dimensional engineered bone from bone marrow stromal cells and their autogenous extracellular matrix. Tissue Eng A 2009;15:187-195.
30. Laurencin CT, Freeman JW. Ligament tissue engineering: An evolutionary materials science approach. Biomaterials 2005;26:75307536.

31. Laurent CP, Durville D, Mainard D, Ganghoffer JF, Rahouadj R. A multilayer braided scaffold for anterior cruciate ligament: Mechanical modeling at the fiber scale. J Mech Behav Biomed Mater 2012;12:184-196.

32. Laurent CP, Ganghoffer JF, Babin J, Six JL, Wang X, Rahouadj R. Morphological characterization of a novel scaffold for anterior cruciate ligament tissue engineering. J Biomech Eng 2011;133: 065001.

33. Lam CX, Hutmacher DW, Schantz JT, Woodruff MA, Teoh SH. Evaluation of polycaprolactone scaffold degradation for 6 months in vitro and in vivo. J Biomed Mater Res A 2009;90:906-919.

34. Leong NL, Kabir N, Arshi A, Nazemi A, Wu B, Petrigliano FA, McAllister DR. Evaluation of polycaprolactone scaffold with basic fibroblast growth factor and fibroblasts in an athymic rat model for anterior cruciate ligament reconstruction. Tissue Eng A 2015;21: 1859-1868.

35. Owaki T, Shimizu T, Yamato M, Okano T. Cell sheet engineering for regenerative medicine: Current challenges and strategies. Biotechnol J 2014;9:904-914.

36. Liu Y, Ramanath HS, Wang DA. Tendon tissue engineering using scaffold enhancing strategies. Trends Biotechnol 2008;26:201-209.

37. Sahoo S, Ouyang H, Goh JC, Tay TE, Toh SL. Characterization of a novel polymeric scaffold for potential application in tendon/ligament tissue engineering. Tissue Eng 2006;12:91-99.

38. Vaquette C, Kahn C, Frochot C, Nouvel C, Six JL, De Isla N, Luo LH, Cooper-White J, Rahouadj R, Wang X. Aligned poly(l-lacticco-e-caprolactone) electrospun microfibers and knitted structure: A novel composite scaffold for ligament tissue engineering. J Biomed Mater Res A 2010;94:1270-1282.

39. Hoyer M, Meier C, Breier A, Hahner J, Heinrich G, Drechsel N, Meyer M, Rentsch C, Garbe LA, Ertel W, Lohan A, Schulze-Tanzil G. In vitro characterization of self-assembled anterior cruciate ligament cell spheroids for ligament tissue engineering. Histochem Cell Biol 2015;143:289-300.

40. Altman GH, Horan RL, Lu HH, Moreau J, Martin I, Richmond JC, Kaplan DL. Silk matrix for tissue engineered anterior cruciate ligaments. Biomaterials 2002;23:4131-4141.

41. Freeman JW, Woods MD, Laurencin CT. Tissue engineering of the anterior cruciate ligament using a braid-twist scaffold design. J Biomech 2007:40:2029-2036.

42. Cao D, Liu W, Wei $X, X u$ F, Cui L, Cao Y. In vitro tendon engineering with avian tenocytes and polyglycolic acids: A preliminary report. Tissue Eng 2006;12:1369-1377.

43. Hairfield-Stein M, England C, Paek HJ, Gilbraith KB, Dennis R, Boland E, Kosnik P. Development of self-assembled, tissue-engineered ligament from bone marrow stromal cells. Tissue Eng 2007:13:703-710.

44. Mitani G, Sato M, Yamato M, Kokubo M, Takagaki T, Ebihara G, Okano T, Mochida T. Potential utility of cell sheets derived from the anterior cruciate ligament and synovium fabricated in temperatureresponsive culture dishes. J Biomed Mater Res A 2014;102:29272933.

45. Calve S, Lytle IF, Grosh K, Brown DL, Arruda EM. Implantation increases tensile strength and collagen content of self-assembled tendon constructs. J Appl Physiol 2010;108:875-881. 